\title{
Integrated Behavioral Health Model for COVID-19 Patients
}

Ronald $R$ O'Donnell, $P h D^{1 *}$, Shiyou Wu, MSW, PhD', Jennifer Rolfes, MS (Statistics), DBH, MBA3 , Patraporn Tungpunkom, PhD, MSN, APPMH, RN, FAAN (Co-I) and Hoang D Nguyen, PhD 5

${ }^{1}$ Clinical Professor, College of Health Solutions, Arizona State University, USA

${ }^{2}$ Assistant Professor, School of Social Work, Arizona State University, USA

${ }^{3}$ Chief Data and Research Officer, Cornerstone Whole Healthcare Organization, Inc, USA

${ }^{4}$ Associate Professor \& Director, The Thailand Center for Evidence-Based Health Care, Faculty of Nursing, Chiang Mai University, Thailand

${ }^{5}$ Assistant Professor, School of Computing Science, University of Glasgow, Singapore

\section{Background}

The COVID-19 pandemic has impacted health outcomes for noncommunicable diseases (NCD's), lifestyle behaviors, psychiatric and substance use disorders in two ways. First, individuals infected with COVID-19 who have comorbid NCD's, lifestyle risk factors and psychiatric conditions are at greater risk for morbidity and mortality than individuals without these comorbidities, and many suffer ongoing physical symptoms and/or psychiatric problems [1]. Second, the population at large is responding to the COVID-19 pandemic with increased depression, anxiety, and unhealthy lifestyle behaviors that are attributed to fear of COVID-19 infection, stress associated with job losses and reduced income, social distancing and lockdown measures [2]. The adverse impact of COVID-19 on medical, lifestyle and psychiatric conditions points to a need for an integrated behavioral approach that systematically addresses lifestyle habits underlying NCD onset and progression and the psychiatric problems for patients infected and for those at risk of infection [2].

For the population at risk of contracting COVID-19, reducing NCD risk factors, improving lifestyle habits, and treating depression, anxiety and other problems will reduce the risk of adverse outcomes if infected with COVID-19 [3]. The population at large needs an approach to address the psychological consequences of COVID-19 [4]. An integrated behavioral health approach to NCD's, lifestyle and psychiatric problems delivered via telehealth and enhanced with other health technologies such as mHealth (smartphone apps), wearables and remote monitoring is needed in response to the COVID-19 pandemic.

COVID-19 patients with NCD's such as diabetes, hypertension, and cardiovascular disease are at greater risk of complication and death [5]. Lifestyle health behaviors and obesity contribute to NCD's and poor outcomes for COVID-19 patients [3]. Optimal nutrition status to strengthen the immune system has been used as a measure of resilience towards stabilization during the COVID-19 pandemic [6]. Healthy eating may be adversely impacted by the stress of COVID-19 lockdown, social isolation and greater difficulty accessing healthy foods [7]. Physical exercise is recommended as a therapy to prevent adverse physical and psychological consequences of the COVID-19 quarantine, especially among the elderly [8].

In addition, a public health survey in China found that $53.8 \%$ of respondents rated the psychological impact of the pandemic as moderate to severe [1]. COVID-19 patients with mental health disorders face more barriers to access to care due to stigma and discrimination in health care settings [9] and comorbid mental health disorders may result in less effective medical treatment [10].

\section{Integrated Behavioral Health}

Integrated behavioral health is a patient-centered, teambased and coordinated approach to treating NCD's, lifestyle behaviors, psychiatric and substance use disorders in primary care. It is also a major component of primary healthcare reform in the United States [11]. In practice, an expert behavior-

*Corresponding author: Ronald $\mathrm{R}$ O'Donnell, PhD, Clinical Professor, College of Health Solutions, Arizona State University, USA

Accepted: January 21, 2021

Published online: January 23, 2021

Citation: O'Donnell RR, Wu S, Rolfes J, et al. (2021) Integrated Behavioral Health Model for COVID-19 Patients. Arch Fam Med Gen Pract 6(1):169-173 
al health consultant $(\mathrm{BHC})$ on the primary care team provides interventions for management and prevention of NCD's, behavioral medicine for lifestyle problems, and behavioral interventions for comorbid psychiatric and substance use disorders, and team-based consultation and quality improvement [12]. The BHC is specially trained in behavioral interventions such as improved nutrition and physical activity, smoking cessation and reduced harmful alcohol use [13]. Integrated behavioral health includes a population health management approach to prevent and manage NCD's through screening, stratify based on problem severity, provide evidence-based and stepped-care interventions tailored to patient risk, and systematic follow-up [14]. The use of digital health technologies also helps to promote patient engagement and self-management of health behaviors [15] (Figure 1).

\section{Recommendations: An Integrated Behavioral Health Model for COVID-19}

There is a critical need to address chronic disease, behavioral health, and health behaviors when determining COVID-19 risk, severity, and prioritization of services. Lindner, et al. [2] recommend a multi-staged process that utilizes health technologies to complete remote triage, telehealth and remote monitoring of COVID-19 patients. Other recommendations include quarantine or general home-health services including remote contact with lifestyle coaches, athome physical activity, and case manager follow-up to pro- vide personalized care planning [2]. Bakshi, et al. [16] (in press) report a COVID-19 population health program that includes identification of high-risk patients and potential gaps in care, care management and home-based services, behavioral health interventions and telemedicine.

The recommendation by this group is a COVID-19 integrated behavioral health program delivered by a BHC in consultation with the medical team and specialty care providers. A population health management approach will be used to conduct a COVID-19-related patient risk stratification using a Health Risk Assessment (HRA) comprised of domains that reflect common mental health, substance abuse, health behaviors and pandemic behaviors specific to COVID-19 and related infectious diseases. The goal is to identify diagnoses (e.g., COVID-19, and other NCD's), lifestyle (e.g., nutrition, physical activity), psychological (e.g., anxiety, depression) demographic and Social Determinants of Health (e.g., income, marital and employment status, and health literacy) in patients to target treatment based on a profile of COVID-19 risk factors.

Recommendations for the HRA include patient self-report measures from My Own Health Report (MOHR) [17] and additional screening measures relevant to COVID-19. The MOHR domains are nutrition, physical activity, risky alcohol use, smoking, anxiety, depression, stress, and sleep. The following COVID-19 related screening measures are also recommended: The Somatic Symptom Scale for common physical

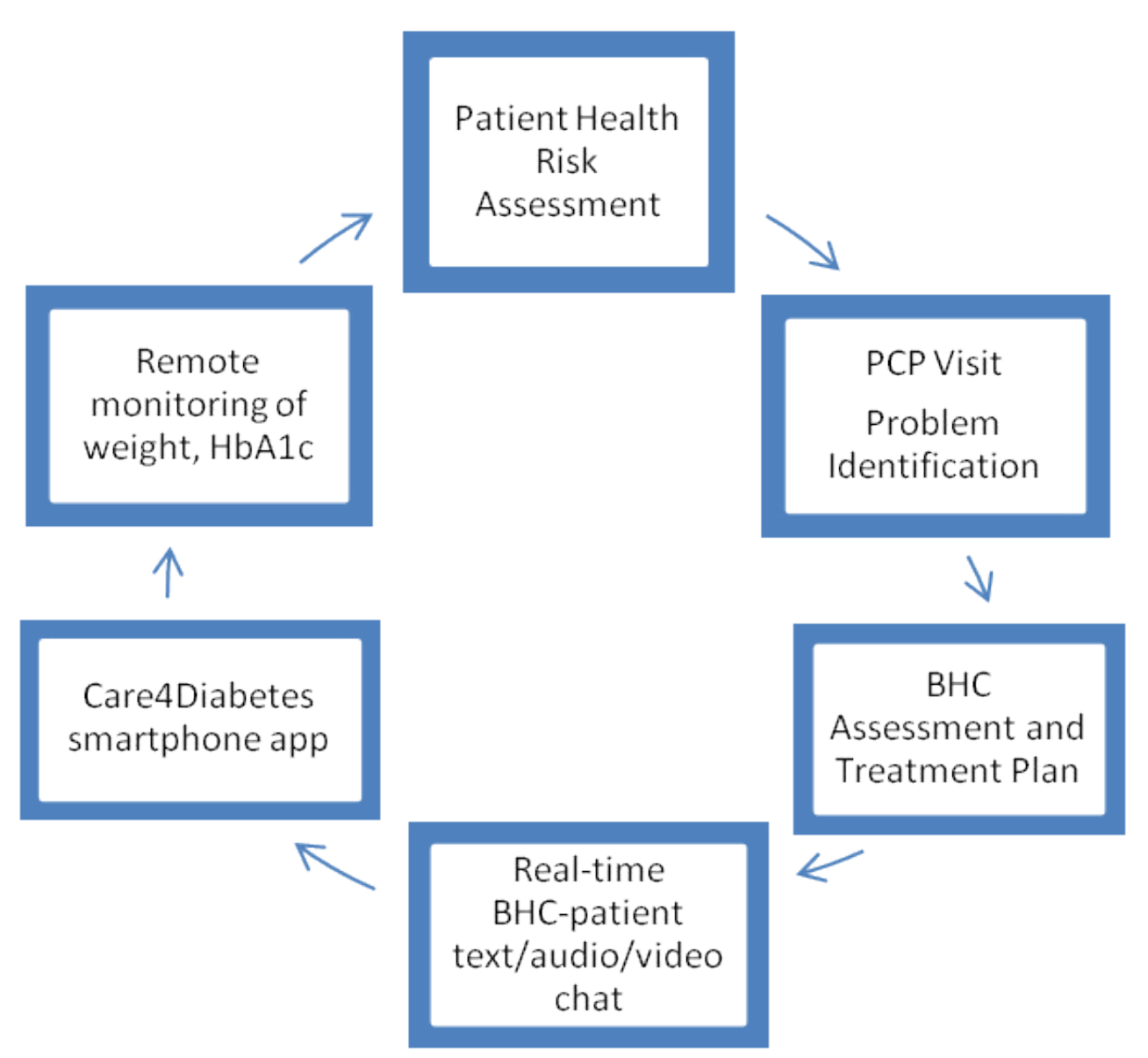

Figure 1: Integrated behavioral health model. 
symptoms [18]; the Abbreviated PTSD Civilian Checklist [19]; the Morisky Medication Adherence Scale [20] to assess medication adherence; the Quality of Life [21], and domestic violence [22].

Studies of smart phone app use to improve diabetes self-management show an overall reduction of $8 \%$ in $\mathrm{HbA} 1 \mathrm{c}$ for type 2 diabetes myelitis (T2DM) compared to usual care [23]. An example of a smartphone app Care4Diabetes [24] that is designed to support patient self-management of life- style behaviors for T2DM will also address COVID-19-related health risks. The Care4Diabetes app includes the following content: education sessions, exercise, dietary logs, medication/insulin logs, blood glucose monitoring, automatically individualized reminder, and telephone/video conference [24]. The strength of the Care4Diabetes app is the diabetes-specific content and an interactive nurse portal for seamless communication between patient and clinician. The nurse portal is essential for remote care management in the context of barri-
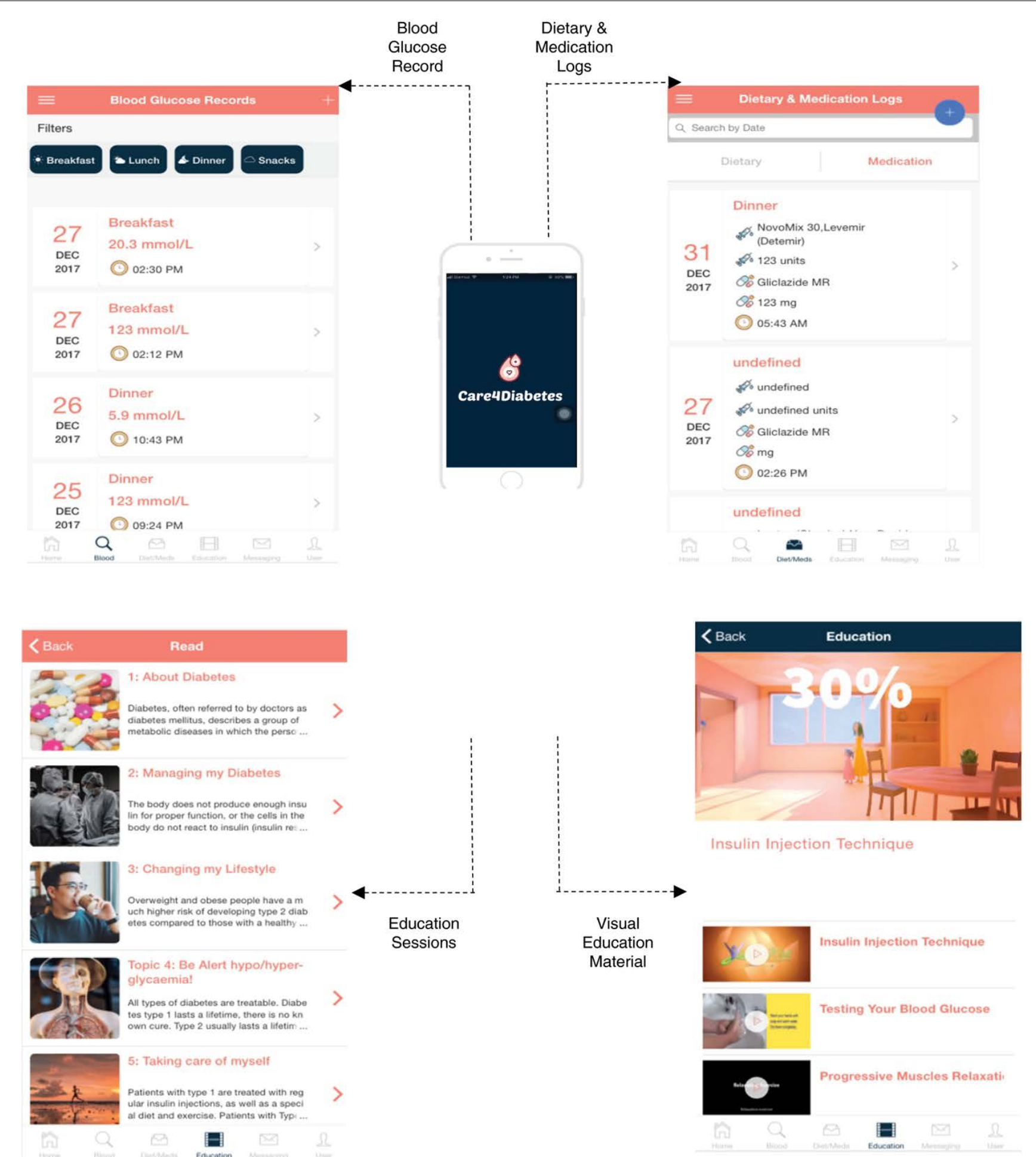

Figure 2: Care4Diabetes App. From Wang, et al. [1]. 
ers to in-person care during COVID-19. This app will facilitate safer online self-management of patient health behaviors during the current COVID-19 pandemic. In a recent study patients shared that the Care4Diabetes app was easy to use, especially to update their blood glucose, diet and medications [5]. Recent studies have recommended remote telehealth for COVID-19 patients for assessment [25] and for mental health problems [26] in order to reduce the risk of clinic-based infection (Figure 2).

COVID-19 patients discharged to home and the entire population at large during a pandemic will benefit from remote screening, assessment and treatment planning that reduces the risk of contracting the virus. T2DM patients who are not infected with COVID-19 will benefit from improved health behaviors and reduced mental health problems as protective factors for COVID-19. Patients' health data related to COVID-19 comorbidities are collected and reviewed in real time by the BHC, patients and their family members/caregivers. Patients can review charts or graphs on physical activity, and self-reported nutrition and other patient reported outcomes (e.g., depression, stress) and their motivation based on readiness to change.

A workforce education and training component is needed for BHC's, physicians, nurses and community health workers in primary care and hospital settings. Clinical social workers are an example of the type of workforce needed to serve as BHC's on the primary care team [27]. The clinician training will include how to review the HRA results with the patient using the 5 A's (Assess, Advise, Agree, Assist, Arrange) approach to completing a health assessment and shared decision-making. Lifestyle interventions for nutrition and physical activity will be utilized to improve resilience as protective factors for COVID-19. Patients will be encouraged to monitor their HRA results at home and to consult with the clinician remotely by phone or SMS text messaging. The role of the BHC is to provide the oversight to evaluate the patient progress towards health behavior self-management over time, to identify and address barriers to care, and consult and coordinate care with the primary care or hospital team.

\section{Summary}

The integrated behavioral approach appears ideally suited to managing the combined health risks of COVID-19, NCD's, lifestyle behaviors, psychiatric and substance use disorders. The key components of integrated behavioral health for COVID-19 include a BHC as the key point of contact, working in collaboration with the at-risk patient to review health risks, agree and implement behavior change interventions, and provide systematic follow-up. Population health management strategies and health technologies are incorporated based on evidence for effectiveness and the need to provide care remotely due to COVID-19-related barriers to in-person care.

\section{References}

1. Wang B, Li R, Lu Z, et al. (2020) Does comorbidity increase the risk of patients with COVID-19: Evidence from meta-analysis. Aging 12: 6049-6057.
2. Lindner S, Kubitschke L, Lionis C, et al. (2020) Can integrated care help in meeting the challenges posed on our health care systems by COVID-19? Some preliminary lessons learned from the European VIGOUR project. Int J Integr Care 20: 4.

3. Sheldon TA, Wright J (2020) Twin epidemics of covid-19 and non-communicable disease. BMJ 369.

4. Ho CS, Chee CY, Ho RC (2020) Mental health strategies to combat the psychological impact of COVID-19 beyond paranoia and panic. Ann Acad Med Singapore 49: 155-160.

5. Wang W, Cheng MTM, Leong FL, et al. (2020) The development and testing of a nurse-led smartphone-based self-management programme for diabetes patients with poor glycaemic control. Journal of Advanced Nursing 76: 3179-3189.

6. Aman F, Masood S (2020) How Nutrition can help to fight against COVID-19 Pandemic. Pak J Med Sci 36(COVID19-S4): S121-S123.

7. Naja F, Hamadeh R (2020) Nutrition amid the COVID-19 pandemic: A multi-level framework for action. Eur J Clin Nutr 74: 11171121.

8. Jiménez-Pavón D, Carbonell-Baeza A, Lavie CJ (2020) Physical exercise as therapy to fight against the mental and physical consequences of COVID-19 quarantine: Special focus in older people. Prog Cardiovasc Dis 63: 386-388.

9. Yao H, Chen J, Xu Y (2020) Patients with mental health disorders in the COVID-19 epidemic. Lancet Psychiatry 7: e21.

10. Norman Sartorious (2013) Comorbidity of mental and physical diseases: a main challenge for medicine of the 21st century. Shanghai Arch Psychiatry 25: 68-69.

11. Manderscheid R, Kathol R (2014) Fostering sustainable, integrated medical and behavioral services in medical settings. Ann Intern Med 160: 61-65.

12. Vogel ME, Kanzler KE, Aikens JE, et al. (2017) Integration of behavioral health and primary care: Current knowledge and future directions. J Behav Med 40: 69-84.

13. Reiter JT, Dobmeyer AC, Hunter CL (2018) The primary care behavioral health $(\mathrm{PCBH})$ model: An overview and operational definition. J Clin Psychol Med Settings 25: 109-126.

14. Hodach R, Grundy P, Jain A, et al. (2016) Provider-led population health management: Key strategies for healthcare in the cognitive era. John Wiley \& Sons.

15. Raney L, Bergman D, Torous J, et al. (2017) Digitally driven integrated primary care and behavioral health: How technology can expand access to effective treatment. Curr Psychiatry Rep 19: 86.

16. Bakshi S, Schiavoni K, Carlson L, et al. (2020) The essential role of population health during and beyond COVID-19. Am J Manag Care 27.

17. Glasgow R, Kessler R, Ory M, et al. (2014) Conducting rapid, relevant research: Lessons learned from the my own health report project. Am J Prev Med 47: 212-219.

18. Toussaint AM, Murray A, Voigt K, et al. (2016) Development and validation of the somatic symptom disorder-B criteria scale (SSD12). Psychosom Med 78: 5-12.

19. Lang A, Stein M (2005) An abbreviated PTSD checklist for use as a screening instrument in primary care. Behav Res Ther 43: 585-594.

20. Morisky DE, Green LW, Levine DM (1986) Concurrent and pre- 

6(1):169-173

dictive validity of a self-reported measure of medication adherence. Medical Care 24: 67-74.

21. Vahedi S (2010) World Health Organization Quality-of-Life Scale (WHOQOL-BREF): Analyses of their item response theory properties based on the graded responses model. Iranian J Psychiatry 5: 140-153.

22. Davis JW, Parks SN, Kaups KL, et al. (2003) Victims of domestic violence on the trauma service: Unrecognized and underreported. J Trauma 54: 352-355.

23. Kitsiou S, Pare G, Jaana M, et al. (2017) Effectiveness of mHealth interventions for patients with diabetes: An overview of systematic reviews. PLoS One 12: e0173160.
24. Wang W, Seah B, Jiang Y, et al. (2018) A randomized controlled trial on a nurse-led smartphone-based self-management programme for people with poorly controlled type 2 diabetes: A study protocol. J Adv Nurs 74: 190-200.

25. Greenhalgh T, Koh GCH, Car J (2020) Covid-19: A remote assessment in primary care. BMJ 368.

26. Liu S, Yang L, Zhang C, et al. (2020) Online mental health services in China during the COVID-19 outbreak. The Lancet Psychiatry 7: e17-e18.

27. Fraser MW, Lombardi BM, Wu S, et al. (2018) Integrated primary care and social work: A systematic review. Journal of the Society for Social Work and Research 9: 175-215. 